\title{
A new class of quantum bound states: diprotons in extreme magnetic fields
}

\author{
Danielle Allor, Paulo Bedaque and Thomas D. Cohen \\ Department of Physics \\ University of Maryland \\ College Park, MD 20742 \\ Charles T. Sebens \\ Department of Physics \\ Massachusetts Institute of Technology \\ Cambridge, MA 02139
}

\begin{abstract}
This paper considers the possibility that two charged particles with an attractive short-ranged potential between them which is not strong enough to form bound states in free space, may bind in uniform magnetic fields. It is shown that in the formal limit where Coulomb repulsion is negligible ( $q \rightarrow 0$ and $B_{0} \rightarrow \infty$ with $q B_{0}$ fixed, where $q$ is the charge and $B_{0}$ the field strength) there always exists a bound state for a system of two identical charged particles in a constant magnetic field, provided that there is a short-range uniformly attractive potential between them. Moreover, it is shown that in this limit any potential with an attractive s-wave scattering length will posses bound states provided that the range of the potential is much smaller than the characteristic magnetic length, $r_{0} \equiv\left(\frac{q B_{0}}{4}\right)^{-1 / 2}$. For this case, the binding is computed numerically. We estimate the size of the magnetic field needed to approximately reach a regime where the formal limit considered becomes a good approximation to the dynamics. These numerical estimates indicate that two protons in an extremely strong magnetic field such as might be found in a magnetar will bind to form a diproton.
\end{abstract}

\section{INTRODUCTION}

In nonrelativistic quantum mechanics, uniformly attractive potentials in one or two dimensions always allow the existence of bound states. While this is not generally true in three dimensions, the presence of a magnetic field can alter the situation considerably: attractive potentials between two charged particles with insufficient strength to bind in free space can bind in the presence of a strong external magnetic field. This novel class of quantum bound states is the focus of the present paper.

Consider the nonrelativistic quantum dynamics of two charged particles in a uniform magnetic field. It is useful to specialize to the case where the charge and mass of the two particles are identical; in this situation the center-ofmass motion decouples from the relative motion. We begin the analysis working in a formal limit:

$$
q \rightarrow 0 \text { and } B_{0} \rightarrow \infty \text { with } q B_{0} \text { fixed. }
$$

This limit has the property that the interaction between each particle and the external magnetic remains of fixed strength while the Coulomb repulsion between the particles is suppressed.

As a first example we consider a finite-ranged centralforce potential between the two particles which is attractive at all distances but is not strong enough to bind the particles in the absence of the magnetic field. In such circumstances there is a general theorem that the two particles will necessarily have a bound state even if the potential is not strong enough to bind in free space. However, the emergence of bound states in magnetic fields from potentials not strong enough to bind in free space is more general than this. One important case where such binding is guaranteed to happen in the limit $q \rightarrow 0$ and $B_{0} \rightarrow \infty$ with $q B_{0}$ fixed is when there is a finite ranged interaction with both attractive and repulsive regions but which has net attraction at low energies in the sense that the scattering length, $a$, is attractive and the characteristic magnetic length scale $\left(\frac{q B_{0}}{4}\right)^{-1 / 2}$ (where we work in natural units natural units of $\hbar=c=1$ ) is much larger than the range of the interaction $R$.

There are simple intuitive reasons why such binding might be expected. Consider first classical scattering in the situation described. Qualitatively, if the Coulomb repulsion is neglected, two non-interacting particles with the same charge and mass moving in a magnetic field will have the same angular frequency. Thus after they scatter they will bend in the same manner and thereby remain close to one another for a longer time than an analogous scattering in free space. This in turn allows the attractive but finite-ranged interaction to remain significant for a longer period leading to increased net attraction. The quantum analog of this is presumably also increased attraction compared to the free space case and it is not surprising that this can bring about binding.

It can also be understood qualitatively from a quantum mechanical perspective. Recall that a charged particle in a constant magnetic field of strength $B_{0}$ moves at the cyclotron frequency $\omega_{c}=\frac{q B_{0}}{m}$ with an energy spectrum described by the well-known Landau levels. This can be easily seen via an appropriate choice of gauge (the symmetric gauge) in which case the Hamiltonian of a single charged particle in a magnetic field can be written as the sum of a two-dimensional harmonic oscillator Hamiltonian and an angular momentum term in the direction of the magnetic field. In this gauge, the magnetic field (which without loss of generality we take to be in the $\hat{z}$ 
direction) acts to restrict the particles to confined harmonic motion in the $x-y$ plane, leaving free particle motion only along the direction of the magnetic field. Under these circumstances, it is highly plausible that a situation similar to the one dimensional case might arise in which any purely attractive potential between the two particles will generically bind them.

Given the generality of the result, one might think that such bound states should be ubiquitous. However, there is a fundamental limitation: the analysis was carried out in the regime $q \rightarrow 0$ and $B_{0} \rightarrow \infty$ with $q B_{0}$ fixed. In practice, however, one cannot find particles with arbitrarily small charges since the charge is quantized in units of $e$. Thus to approach the regime of relevance one needs to find situations in which the Coulomb interaction in the lowest lying state is very small compared to the characteristic energies in the problem arising from the particles interacting with the magnetic field, (i.e. the cyclotron frequency $\left.-\omega_{c}=\frac{q B_{0}}{m}\right)$ and is also much smaller than the characteristic energy scale of the short-ranged attraction between the particles. In practice, these conditions are hard to obtain. One requires very large magnetic fields.

The requirement of extreme magnetic fields implies that the effect in its simplest form will not be directly relevant for systems of atomic or molecular ions. Note that, although one can describe the interactions between such ions quantum mechanically via an effective potential, the potential depends on the internal structure of the ions. The internal structure will be greatly altered due polarization effects in very strong fields. Thus one cannot describe the interaction of the two ions in the presence of the magnetic field as essentially the free space interaction. Of course, there may well be situations in which two atomic or molecular ions bind in the presence of a magnetic field which do not bind in free space. However, such an effect would be a many-body effect in which the effective two-body potential between the atoms is altered due to the changed internal structure. This is not the effect discussed here. As we will show, the magnetic fields needed to see the phenomenon discussed here are many orders of magnitude larger than the characteristic field strength needed to alter the interparticle potential by altering the internal structure

Thus, the most natural place to look for the effect is where strong interactions dominate -i.e. in nuclear physics. The most favorable case we know is for bound states of the two protons. As we will discuss below, such diprotons are expected to form in magnetic fields of $10^{12} \mathrm{~T}$. Such fields are found in nature but static fields of this scale are only seen in astrophysical contexts such as magnetars. It remains an open question as to whether there are any astrophysical consequences of these states which have direct implication for observation. Whether this turns out to be the case or not, these states are interesting quantum mechanical curiosities in their own right.

Before proceeding it is useful to define what we mean by a bound state in this context. In free space this is unambiguous: the relative and center of mass motion decouple, and bound states have a discrete energy spectrum, negative energy and a normalizable wave function for the relative motion; the probability of finding the particles at some fixed distance drops off exponentially with distance. In contrast, continuum states in free space have a continuum spectrum, positive energy and nonnormalizable states. In a magnetic field things are dramatically different. In the first place, relative and centerof-mass motion do not generally decouple. Secondly, the distinction between discrete and continuum spectra becomes ambiguous: even non-interacting single particles have a spectrum with discrete Landau levels (with infinite degeneracy) along with a continuum index. Given this situation, we begin as a technical matter with a definition of a "bound state" in terms of the behavior of the drop off of the probability amplitude with distance: $a$ bound state is an energy eigenstate in which the probability amplitude for finding two particles a distance $r$ apart falls of at long distances faster than a power law in all directions. This definition is, of course, fully consistent with zero-field case and is a useful generalization.

In this paper, we restrict our attention to situations in which the charge and mass of the two particles are identical. As will be discussed below, provided that the dynamics are non-relativistic, the center-of-mass and the relative motion cleanly separate in this case. The centerof-motion behaves as a single particle of mass $M=2 \mathrm{~m}$ and charge $Q=2 q$; it has a spectrum of (infinitely degenerate) discrete Landau levels plus a continuum associated with the motion in the $\hat{z}$ direction. Bound states in this circumstance have two properties analogous to the free space case: i) the wave functions for the relative motions are normalizable and ii) the energy of the state is negative by a non-zero amount relative to what it would be if the particles were noninteracting or equivalently were very far apart.

The goal of this paper is to explore the phenomenon of binding due to intense magnetic fields rather generally. We will show that in certain circumstances the phenomenon must occur. However, for any particular interaction whether or not binding occurs and, if it does, the value of the binding energy requires a detailed knowledge of the interaction and a numerical solution of the Schrodinger equation. It is useful to try to understand how this effect occurs rather generally without relying on too many details of the interaction (which in many cases is not known with precision in any event). Accordingly, we focus here on some limiting cases through which one can gain insight into the phenomenon without specifying the details of the particle-particle interaction. The key limitations in this analysis are a restriction to situations in which the Coulomb interaction may be neglected and where the magnetic fields are small enough so that the characteristic magnetic length scale is much longer than the range of the potential. While these limitations are severe, there is at least one interesting physical systema bound state of protons in magnetar strength fields - for which the approximations appear to be quite good. 
The plan of the paper is as follows: We begin by setting up the general problem and then prove that, in a constant magnetic field and in the limit $q \rightarrow 0$ and $B_{0} \rightarrow$ $\infty$ with $q B_{0}$ fixed, two charged particles with the same charge and mass and with a uniformly attractive potential between them always have a bound state. We also prove that in this regime any potential which is attractive in the sense of having an attractive scattering length must have a bound state in a sufficiently weak magnetic field and derive an analytic expression for the binding energy for weak magnetic fields. In connection to this weak field limit we note that it is very difficult to simultaneously have sufficiently weak fields to be in this regime and to have the Coulomb repulsion be small enough to neglect.

Next we consider systems for which the scattering length is "unnatural", ie. much larger then the characteristic range of the interaction. In this regime there is an effective field theory [1, 2] describing the system and one can compute binding without a detailed microscopic knowledge of the interaction. This regime is of phenomenological interest in low energy nuclear physics since the nucleon-nucleon scattering length is much larger than the characteristic range of the nuclear force. We numerically calculate the binding energy of the bound state in this effective theory as a function of a dimensionless parameter proportional to $a B_{0}^{1 / 2}$ where $a$ is the scattering length and $B_{0}$ is the strength of the magnetic field. We discuss corrections to this result both due to Coulomb repulsion and to short-distance effects. For the case of two protons we use dimensional arguments to argue that a regime exists in which both of these corrections are simultaneously moderately small.

Following this we discuss the case where the two particles have spin. There is a general argument that energy of interaction of the spins with the magnetic field are of the same order as the binding effects considered here. It is easy to see that in the regime of validity of the effective theory the spin degrees of freedom decouple from the spatial ones. This means that the bound states considered here will continue to exist. However, in the case of identical fermions it may well happen that even when there is a bound state of the sort discussed in this paper, the ground state of the system need not be bound. This can come about because the Pauli principle combined with the dynamics of the bound state can require the spins to be in distinct $m$-states. The energy gained in binding can be less than the energy lost by aligning the spins in the field. This situation is radically different from what occurs in free space where the ground state must be bound if any bound states exist. As we will see later, the situation where the lowest bound state is not the ground state is precisely the circumstance for the diproton.

We end the paper with a brief concluding section including a few words about the possible astrophysical significance.

\section{BOUND STATES IN MAGNETIC FIELDS}

\section{A. Generalities}

In this section we set up the general problem and prove the existence of a bound state when there is a potential which is attractive everywhere between two identical charged particles in a constant magnetic field in the limit $q \rightarrow 0$ and $B_{0} \rightarrow \infty$ with $q B_{0}$ fixed.

Consider the case of two charged nonrelativistic particles (with the same mass $m$ and the same charge $q$ ) interacting via a central potential. For simplicity we will neglect spin effects here and return to the question of spin later in the paper; accordingly we will assume a central potential initially. For simplicity we will assume that the two particles have identical charges and masses. The Hamiltonian of the system is then

$$
\hat{H}=\frac{\left(p_{1}-q \hat{A}\right)^{2}}{2 m}+\frac{\left(p_{2}-q \hat{A}\right)^{2}}{2 m}+V\left(\left|\vec{r}_{1}-\vec{r}_{2}\right|\right) .
$$

Without loss of generality we can take the magnetic field to be in the $\hat{z}$ direction. We find it convenient to work with the symmetric gauge

$$
\vec{A}=\frac{B_{0}}{2}(x \hat{y}-y \hat{x}) .
$$

For the case of interest here where the mass and charge of the two particles are equal, the center-of-mass and relative motion separate. With $\mu=\frac{m}{2}$ as the reduced mass and $M=2 m$ as the total mass, the relative and center-of-mass Hamiltonians in configuration space are:

$$
\begin{gathered}
\hat{H}_{r e l}=-\frac{1}{2 \mu} \nabla^{2}+\frac{1}{2} \mu \omega^{2}\left(x^{2}+y^{2}\right)-\omega L_{z}+V(|r|) \\
\hat{H}_{C o M}=-\frac{1}{2 M} \nabla^{2}+\frac{1}{2} M \omega^{2}\left(x^{2}+y^{2}\right)-\omega L_{z}
\end{gathered}
$$

where $\omega=\frac{1}{2} \omega_{c}=\frac{q B_{0}}{M}=\frac{q B_{0}}{4 \mu}$.

In the absence of a short-range potential, the center of mass and the relative equations closely resemble the Hamiltonian of a single particle with a Landau level spectrum. In the absence of interactions between the particles the relative motion does as well. Therefore, without the potential, the total energy for the ground state of the relative motion is $\omega=\frac{q B_{0}}{4 \mu}$. A bound state corresponds to an energy below this value.

It is easy to convince one's self that to minimize the energy the term proportional to $L_{z}$ in Eq. (2.3) should be zero. It is always possible to do this since $L_{z}$ commutes with the Hamiltonian. With the choice of gauge of Eq. (2.2) the Hamiltonian can be written:

$$
\hat{H}_{r e l}=-\frac{1}{2 \mu} \nabla^{2}+\frac{1}{2} \mu \omega^{2} \rho^{2}-V(\vec{r}),
$$

where $V(\vec{r})$ is the potential, and $\nabla^{2}$ is the three dimensional Laplacian. A two-dimensional harmonic oscillator 
term, $\hat{H}_{2 D-H O}$, may be isolated from Eq. (2.5) and the Hamiltonian may be rewritten as follows:

$$
\hat{H}_{r e l}=\hat{H}_{2 D-H O}-\frac{1}{2 \mu} \frac{\partial^{2}}{\partial z^{2}}-V(\vec{r}) .
$$

\section{B. A theorem}

A variational argument may then be used to show that a uniformly attractive potential always binds with the Hamiltonian in Eq. (2.6). Before doing this note that the condition in Eq. (1.1) is implicit in taking a uniformly attractive interaction; there will always be a long ranged repulsive Coulomb potential away from this limit. To proceed we take a simple a wave function ansätz of

$$
|\psi\rangle=|0\rangle_{2 D-H O}|\phi\rangle_{Z},
$$

where $|0\rangle_{2 D-H O}$ is the ground state of the twodimensional harmonic oscillator in the $x-y$ plane and $|\phi\rangle_{z}$ is an arbitrary state in the Hilbert space for motion in the $z$ direction with $\phi(z)=\langle z \mid \phi\rangle_{z}$; an outer product between is implied. The expectation value of the energy in this state is:

$$
\begin{aligned}
& \left\langle\psi\left|\hat{H}_{\text {rel }}\right| \psi\right\rangle \\
= & \omega+\int d z \phi^{*}(z)\left(-\frac{1}{2 m} \frac{\partial^{2}}{\partial z^{2}}-V_{\text {eff }}(z)\right) \phi(z), \\
& \text { with } V_{\text {eff }}(z) \equiv \int d x d y \psi_{0}^{*}(x, y)(V(\vec{r})) \psi_{0}(x, y)
\end{aligned}
$$

where $\omega$ is the ground state energy of the two-dimensional harmonic oscillator and $\psi_{0}(x, y)$ its normalized wave function. Note that $\psi_{0}^{*} \psi_{0}$ is non-negative; thus, if $V(\vec{r})$ is non-negative for all $\vec{r}$ then $V_{\text {eff }}$ is non-negative for all $z$. That is, a purely attractive $V$ implies a purely attractive $V_{\text {eff. }}$.

Note that the integral in $z$ is the expectation value for the energy of a one-dimensional system with potential $V_{\text {eff. }}$. The effective one-dimensional system must have at least one normalized bound state with negative energy since there is a theorem that all purely attractive one dimensional systems do [3]. Thus, we can choose $\phi(z)$ to be the normalized ground state of this system. In that case we have $\left\langle\psi\left|\hat{H}_{\text {rel }}\right| \psi\right\rangle=\omega-B_{G}^{\text {eff }}$ where $B_{G}^{\text {eff }}$, the binding energy of the effective one-dimensional problem, is positive. Thus we have found a variational state with energy less than that of two non-interacting particles in the magnetic field; the true state must be lower still and thus a bound state must exist.

\section{THE WEAK FIELD LIMIT}

The theorem in the preceding section is of interest as it illustrates the general phenomenon of particles which do not bind in free space forming bound states in magnetic fields. It is limited in that it does not tell us whether a potential which has both attractive and repulsive regions binds in a magnetic field. As a general rule one cannot determine whether this occurs for a particular potential and a given field strength without solving the Schrödinger equation explicitly.

However, there is one general situation where the existence of bound states can be determined by general arguments: the case in which the natural length scale associated with Landau orbits in the magnetic field, $r_{0}=\frac{1}{\sqrt{\mu \omega}}$, is much larger than the range of the potential between the particles (which we will denote as $R$ ). In this weak field situation, it is easy to show that bound states exist providing the scattering length is attractive and Coulomb repulsion is negligible. Moreover, if the scattering length, $a$ is "natural" (i.e. of order $R$ ) and thus much smaller than $r_{0}$, one can directly compute the binding energy. It is given by:

$$
E_{b}=2\left(\frac{a}{r_{0}}\right)^{2} \omega
$$

We note that the weak field condition $r_{0} \gg R$ can be at odds with the regime in which the formal limit $q \rightarrow 0$ and $B_{0} \rightarrow \infty$ with $q B_{0}$ fixed (in which Coulomb repulsion may be neglected) can be regarded as approximately satisfied. The neglect of Coulomb requires $B_{0}$ to be sufficiently large; the condition $r_{0} \gg R$ requires $B_{0}$ to be small. For the problem of the diproton there does exist a window in $B_{0}$ for which the system is in both regimes.

The weak field regime is of interest in that the system is insensitive to the detail of the potential - at leading order, all that is relevant is the scattering length. This is precisely the situation for which an effective field theory [1, 2] is useful. We begin by considering the "natural scattering length" regime of $a \sim R$. We consider the unnatural case $a \gg R$ in a subsequent section.

Our goal is to demonstrate the relationship between the binding energy and the scattering length of Eq. (3.1). We begin with a general expansion of the bound state in a convenient basis:

$$
|\psi\rangle=\sum_{n}|n\rangle_{2 D-H O}\left|\phi_{n}\right\rangle_{z}
$$

where as before an outer product is implied; $\left|\phi_{n}\right\rangle_{z}$ represents the particular $z$ space wave function coupled to a given state of the two dimensional oscillator. The key to our demonstration here is that in the limit $a, R \ll r_{0}$ only the $n=0$ term contributes. We represent the total energy of the system as $\omega$, the energy of the two dimensional harmonic oscillator, minus the quantity of interest: $\hat{H}_{\text {rel }}|\psi\rangle=\left(-E_{b}+\omega\right)|\psi\rangle$. Using the expansion of Eq. (3.2) and multiplying Eq. (2.6) on the left by the 
two dimensional harmonic oscillator state $\langle m(\rho)|$ yields:

$$
\begin{aligned}
\sum_{n}(- & \left.\frac{1}{2 \mu} \frac{\partial^{2}}{\partial z^{2}} \delta_{m n}-V_{m n}(z)\right) \phi_{n}(z) \\
= & \sum_{n}\left(-E_{b}-n \omega\right) \phi_{n}(z) \delta_{m n}
\end{aligned}
$$

where $V_{m n}(z) \equiv \int d \rho 2 \pi \rho \psi_{m}^{*}(\rho) V(\rho, z) \psi_{n}(\rho)$.

Note that the solution to the Schrödinger equation along the $z$-axis decays exponentially according to $e^{-\sqrt{2 \mu E_{b}} z}$ as the binding becomes small. Clearly, the $n=0$ state is the only one which contributes in the weak binding limit because of the $\left(-E_{b}-n \omega\right)$ term; for nonzero $n$, the wavefunction no longer decreases exponentially in the $\hat{z}$ direction as the binding energy becomes very weak. The only term remaining that connects orthogonal $m$ and $n$ states is $V_{m n}$. However, the range of the attractive potential $V$ is small compared to that of the harmonic oscillator. Therefore, all harmonic oscillator states appear essentially flat in the range of the potential, and all contribute approximately equally.

Note that there are now three length scales in the problem: the range of the attractive potential, $R$; the length scale of the harmonic oscillator, $r_{0}=\frac{1}{\sqrt{\mu \omega}}$; and the scale of exponential decay of the wave function in the $\hat{z}$ direction, $\kappa^{-1}=\left(2 \mu E_{b}\right)^{-1 / 2}$. The hierarchy of length scales needed for Eq. (3.6) to be valid is

$$
R \ll r_{0} \ll \kappa^{-1} .
$$

One must test ex post facto to see whether this condition is satisfied. Note that if Eq. (3.1) is correct, then the condition in Eq. (3.4) becomes

$$
R \ll r_{0} \ll r_{0} \frac{1}{a \sqrt{\mu \omega}} .
$$

Eq. (3.3) now takes the form of a one-dimensional Schrödinger equation. Moreover, at small binding the spatial extent of the one dimensional wave function means that the system can be well represented as a system with a Dirac delta potential in $z$ with strength $\alpha$ and energy $-E_{b}$ :

$$
\begin{aligned}
\left(-\frac{1}{2 \mu} \frac{\partial^{2}}{\partial z^{2}}-\alpha \delta(z)\right)\left|\phi_{0}(z)\right\rangle & =-E_{b}\left|\phi_{0}(z)\right\rangle, \\
\text { with } E_{b} & =\frac{\mu \alpha^{2}}{2} .
\end{aligned}
$$

Clearly, to proceed we must determine $\alpha$ in terms of the scattering length.

We know a priori that $\alpha$ does not depend on the details of the short distance potential but only on the scattering length. This suggests a useful strategy for computing $\alpha$ in general, namely to consider any class of potential for which we know how to compute both $\alpha$ and the scattering length and then relate the two. There is a straightforward situation for which we can do this: namely the case of arbitrarily weak potentials. For the bound state problem in a magnetic field this regime gives

$$
\alpha=\int d z 2 \pi \rho d \rho V(\rho, z) \psi_{0}^{*}(0) \psi_{0}(0)
$$

For the scattering, the weak potential limit justifies the the Born approximation. The Born approximation for the scattering length is

$$
a=4 \pi \mu \int d^{3} x V .
$$

Combining Eqs. (3.6), (3.7) and (3.8) yields Eq. (3.1) .

A couple of quick comments about this result are in order. The first is that Eqs. (3.7) and (3.8) only hold for weak potentials. Never-the-less the result in Eq. (3.1) is fully general for $R, a \ll r_{0}$. The reason is clearwe have an effective theory in which the only relevant information about the potential is through the scattering length. How the scattering length arises microscopically is irrelevant. The second is that this result generalizes the theorem considered above. Any potential with attractive scattering length will form a bound state in a magnetic field provided that the system is in a regime in which the Coulomb repulsion is negligible and the range of the potential and its scattering length are much smaller than $r_{0}$.

It is important to note however, that this weak coupling limit is of more theoretical then practical significance. Recall that the analysis depended on two assumptions. The first was that the Coulomb repulsion was negligible and the second that the magnetic length scale is much longer then all scales associated with the potential including the scattering length. Clearly neither assumption is exact and hence there must be corrections to these. One can estimate the size of these corrections.

The characteristic distance between the particles is $\left(\mu E_{b}\right)^{-1 / 2}$ since the system is weakly bound and the wave function is largely beyond the range of the potential. From this one estimates the characteristic scale of the perturbative Coulomb correction to be

$$
\frac{\Delta E_{b}^{\text {Coulomb }}}{E_{b}} \sim \mathcal{O}\left(\frac{q^{2} \mu r_{0}^{2}}{a}\right) \sim \mathcal{O}\left(q^{2}(\mu R) \frac{r_{0}^{2}}{R^{2}}\right)
$$

where $q$ is the charge and in the second form we are taking the scattering length to be of the same order as the range of the potential as one expects for a "natural" (i.e. not fine tuned) problem. It is nontrivial to find a regime for which this is small enough to be considered to be a small correction. In the first place note that the second expression for the ratio of the correction to the dominant part is proportional to $q^{2} \mu R$. For two light nuclei and strong interactions $q^{2} \mu R \sim \frac{Z^{2}}{5 A}$. In comparison, in atomic systems $\alpha \mu R$ is many orders of magnitude larger. As noted earlier this implies that binding of the sort considered in this will not occur in atomic or 
molecular ions. In fact, it is typically marginal at best for nuclear systems in the weak coupling regime. Note that Eq. 3.9) contains a factor of $r_{0}^{2} / R^{2}$. Recall that the weak field regime has $r_{0}^{2} / R^{2} \gg 1$. Thus, there is at best a very small window, and more likely no window at all, for which the system has a natural scattering length and simultaneously is at weak field and has perturbative Coulomb corrections. Fortunately, there are systems with unnatural scattering lengths where binding due to the magnetic field can occur.

\section{UNNATURAL SCATTERING LENGTHS}

In the previous section we considered binding in the regime where both the range of the potential and the scattering length are much smaller than the characteristic magnetic length scale. In this section, a more general case is considered: one in which as before $r_{0} \gg R$ so that the detailed structure of the short-ranged potential remains unimportant but where we no longer require the scattering length to be much shorter than $r_{0}$. Of course, one generally expects that in the absence of fine tuning the scattering length should be of the same scale as the range of the potential (or less in the case of weak potentials). Under such circumstances $r_{0}$ is also much larger than $a$. However, there are systems for which the scattering length is "unnaturally" large, i.e. much larger than the range of the potential. The case of unnatural scattering lengths is critical in nuclear physics since the nucleon-nucleon scattering lengths are unnatural: $a \sim 20 \mathrm{fm}$ while $R \sim 2 \mathrm{fm}$ [4]. Indeed, much of the development of effective field theory methods in nuclear physics in the past decade has concerned the treatment of systems which are unnatural [5, 6, 7, 8]. It is important as a practical matter to consider the regime of unnatural scattering lengths. As noted above the regime considered previously with $r_{0} \gg a$ turns out to be of very limited utility.

To proceed it is useful to reexpress the problem by adding and subtracting a one dimensional harmonic potential in the $\hat{z}$ direction from the Hamiltonian in Eq. (2.3). The Hamiltonian can be rewritten as a three dimensional harmonic oscillator Hamiltonian minus a one dimensional harmonic oscillator potential term in the $\hat{z}$ direction:

$$
\hat{H}_{r e l}=-\frac{1}{2 \mu} \nabla^{2}+\frac{1}{2} \mu \omega^{2} r^{2}-\frac{1}{2} \mu \omega^{2} z^{2}-V(|r|) .
$$

This formulation is convenient for numerical studies in that one can easily implement the calculation in a truncated three dimensional harmonic oscillator basis.

As a first step towards considering the general case with $r_{0} \gg R$ but with the ratio of $r_{0} / a$ arbitrary, we consider the extreme case in which the short range potential in the absence of the magnetic field has a scattering length sufficiently long to be treated as though it were infinite scattering (i.e. as though the system had a zero energy bound state corresponding to the boundary between having bound and unbound states in the absence of a magnetic field). This regime is of interest theoretically in that the potential introduces no scales into the problem - its range is irrelevant since it is so short as to be taken to be zero while the scattering length is irrelevant as it is so long as to be effectively infinite. In such a system the binding energy must be proportional to $\omega$. We wish to find the constant of proportionality.

Clearly, the entire effect of the short-range potential on the wave function for $r_{0} \gg R$ can be encoded as a boundary condition for the s-wave part of the wavefunction at small $r$. One way to see this is to start with a finite ranged potential and consider it in the limit in which the strength of the potential, $V_{0}$, goes to infinity and the range goes to zero in such a manner as to keep the scattering length fixed. Since the range of the potential is taken to zero (to reflect the underlying short range of the potential compared to relevant scales) the boundary condition is at $r=0$. Moreover the zero-range nature of the interaction implies that the boundary condition is insensitive to energy thus the zero energy behavior encoded in the scattering length is sufficient. The condition is

$$
\begin{aligned}
\left.\frac{u_{0}^{\prime}(r)}{u_{0}(r)}\right|_{r=0} & =\frac{1}{a} \\
\text { with } u_{0}(r) & =\frac{r \int d \Omega \psi(r)}{4 \pi}
\end{aligned}
$$

We consider the infinite scattering length case at the outset, so this limiting procedure simply yields the logarithmic derivative of the wave function with respect to $r$ approaching zero at $r=0$. Diagonalizing the Hamiltonian for this system in a truncated basis and testing the numerical convergence of the truncation yields a binding energy of

$$
E_{b}=F_{\infty} \omega \text { with } F_{\infty} \approx .60486
$$

where the result is numerically accurate to the quoted precision. As advertised the binding energy is simply proportional to the spacing of the Landau levels of the free particles, $\omega$.

Of course, an infinite scattering length comes about only through infinite fine tuning. In practice, even when the scattering length is quite large compared to the range of the interaction (as for nucleon-nucleon scattering) it is finite. This can play a significant role since the external magnetic field may be varied which in turn varies $r_{0}$. Thus by changing the external field one can go from the region in which $a \gg r_{0}$ to one in which $a \sim r_{0}$. It is important to consider the situation in which $a \sim r_{0} \gg R$ since this broadens the applicability of the analysis. On general dimensional grounds it is apparent that if one uses $\omega$ to set the scale of the binding energy, then then binding energy can only depend on the dimensionless ra- 


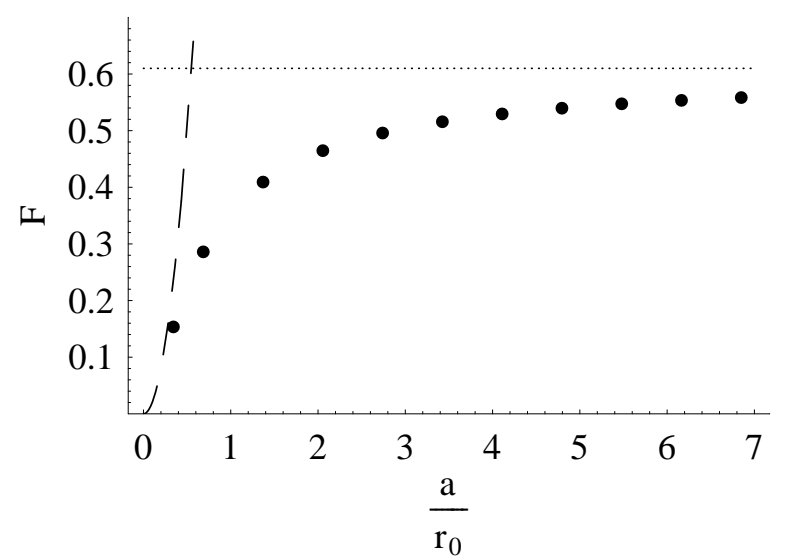

FIG. 1: The ratio of the binding energy to $\omega$ from Eq. (4.4), $\mathrm{F}$, as a function of $a / r_{0}$. The solid dots are the results of numerical calculations. The dotted line indicates the asymptotic value from Eq. (4.3) while the dashed line is the weak field result of Eq (3.1).

tio $a / r_{0}$ :

$$
E_{b}=F\left(a / r_{0}\right) \omega
$$

We know from Eq. (3.1) that for small values $a / r_{0}$, $F\left(a / r_{0}\right) \rightarrow 2 a^{2} / r_{0}^{2}$. Similarly we know from Eq. (4.3) that as $a / r_{0}$ gets large $F$ approaches $F_{\infty} \approx .60486$. The function $F$ can be computed numerically by diagonalizing the magnetic Hamiltonian for the subject to the boundary condition in Eq. (4.2). This is shown in Fig. 1 .

The analysis in this section is more general than the one in Sec. III We have shown that in circumstances where the range of the potential is much shorter than the magnetic length $r_{0}$ one always has a bound state if the scattering length is attractive and if Coulomb repulsion can be neglected, and we have explicitly computed the binding energy in this situation.

Clearly there are errors associated with approximating the potential as zero-ranged and neglecting Coulomb repulsion. If one is in the unnatural regime where $a \sim$ $r_{0} \gg R$ one can estimate the characteristic size of these errors. From general power counting arguments in effective field theory one expects that relative errors induced by treating the potential as having zero range are

$$
\frac{\Delta E_{b}^{\text {finite-range }}}{E_{b}} \sim \mathcal{O}\left(R^{2} / r_{0}^{2}\right)
$$

The relative errors due to the neglect of Coulomb are

$$
\frac{\Delta E_{b}^{\text {Coulomb }}}{E_{b}} \sim \mathcal{O}\left(Q^{2} \mu r_{0} \log \left(R / r_{0}\right)\right)
$$

There is a tension between the two approximations which is seen clearly from Eqs. (4.5) and (4.6). While $\Delta E_{b}^{\text {finite-range }}$ increases with increasing magnetic field,
$\Delta E_{b}^{\text {finite-range }}$ decreases. The optimal value of the magnetic field to minimize both errors will have the two types of errors as being of similar scales. Equating the two yields an error at the optimal field strength for the two approximations of $\Delta E_{b} / E_{b} \sim(\alpha \mu R)^{2 / 3}$. Plugging in values typical for the range of the potential yields an error of order $10 \%$ for the diproton. Thus one expects the analysis done here should have a regime of approximate validity for the diproton case. While it is possible that the corrections are large enough as to yield numerically significant corrections to the result in Fig 1, it seems quite likely that a region does indeed exist for which two protons bind.

The preceding error estimate was done assuming the scattering length is unnatural and of order $r_{0}$. When the scattering length is natural (i.e. $a \sim R$ ) the relative size of the Coulomb correction is much larger and given by Eq. (3.9). Clearly the two results must smoothly interpolate into one another. It is easy to understand qualitatively how this comes about. If one starts with the extreme limit of $a=\infty$ the error estimate in Eq. (4.6) remains valid since then $r_{0}$ sets the full length scale in the problem. As one decreases $a$ with fixed $r_{0}$ two effects occur: the binding energy drops as seen from Fig. 1. This in turn leads to a larger wave functions and hence smaller Coulomb corrections. However, the Coulomb energy drops much more slowly then the binding energy.

Ideally, one would like to calculate this in the regime of validity of an effective field theory treatment-i.e. $r_{0} \gg R, a \gg R$ with $r_{0} / a$ arbitrary. Unfortunately, this can not be done without adding additional information about the system at short distance. If one took seriously our zero-range approximation for the interaction, the perturbative estimate of the Coulomb contribution would diverge logarithmically at short distances due to the singular nature of the Coulomb interaction. Of course, in nature this behavior is regulated by the short distance physics of the interaction. This yields a logarithmic dependence of the ratio of the short distance length associated with the potential and the natural magnetic length scale. Thus, it is apparent that one needs more data about the interaction than merely the scattering length to compute the Coulomb energy. This is unfortunate in that one would like to test the qualitative estimates given in this section to ensure that there are not anomalously large numerical coefficients which might spoil the window where both approximations are reasonably good. We have done simple model calculations to explore this possibility. The models are consistent with our qualitative estimates; none of the models raises doubts about whether two protons bind in the regime where $r_{0} / a \sim .2$.

\section{SPIN AND THE GROUND STATE}

Heretofore we have neglected spin in our discussion. Of course, for the general case particles with non-zero spin, the inter-particle interactions will be spin dependent (e.g. 
there can be a tensor force). As noted previously, for a generic interaction whether or not a magnetic field will yield binding depends on the details of the interaction and must be assessed on a case by case basis through direct simulation. As in the case of spinless particles, if the system is in a regime in which the Coulomb interaction can be neglected and the potential is attractive in all channels and all distances a bound state must occur. The previous variational argument holds with only minor modification. The only new issue is that in setting up a variational ansätz, the state should be put into some particular spin state consistent with the statistics of the problem.

Similarly for $r_{0} \gg R$, the analysis of the previous sections should hold for the energy of a bound state both for the natural scattering length case of Eq. (3.1) and for the unnatural case discussed in Sect. IV] Again, the only new issue is the need to consider a particular spin state consistent with the statistics of the problem. If for example one is studying identical spin $1 / 2$ fermions - as in the case of two protons - either the spin state is symmetric (spin one) and the space is antisymmetric (odd $\mathrm{L}$ ) or the spin state is antisymmetric (spin zero) and the space symmetric (even L). Since we have been focusing on the case where $r_{0} \gg R$ where only the s-wave interaction is relevant the analysis of the previous sections holds provided we restrict our attention to the total spin zero sector. This our previous analysis shows that two protons with total spin zero will form a bound state in sufficiently strong magnetic fields.

However, there is one critical way in which spin can become important in this problem. In free space it is always true that if the two-particle system has a bound state, then the ground state cannot be in the continuum. However, one cannot immediately generalize the intuition gleaned from this fact to the case of bound states in constant magnetic fields. Note that the characteristic scale of the binding energy is of order $\omega$. The interaction energy of the magnetic moments of particles with nonzero spin with an external magnetic field are of the same characteristic order (assuming the g-factors are of order unity). Moreover, for the case of identical spin $1 / 2$ particles these two effects are in competition. The binding effect of the potential lowers the energy for the spin zero state; the magnetic interaction lowers the energy for the spin 1 state (with the moments aligned with the external field). If this magnetic energy turns out to be larger then the ground state is not the bound state.

As it happens, this is precisely the situation for the diproton. The interaction energy of the magnetic moment of the two protons in a spin singlet state is, of course, zero. In the spin aligned state it is:

$$
\Delta E^{\mathrm{spin}-\text { aligned }}=-g B_{0} \mu_{N}=-\frac{g \omega}{2}
$$

where $\mu_{N}$ the nuclear magneton is $\frac{e}{2 m}$. The g-factor for the proton is $g_{p} \approx 5.586$ yielding $\Delta E^{\text {spin-aligned }} \approx$ $-2.793 \omega$. In contrast, one sees from Fig. 1 that the bind- ing energy is bounded from above by $F_{\infty} \omega \approx .60486 \omega$. Thus, the energy the system gained by binding in the spin singlet channel is always more than four times smaller than the energy the system gains by a spin flip into the spin aligned total spin 1 state.

The upshot of this is that although there is rather strong evidence that the diproton has a bound state in sufficiently strong field, the ground state will be in the two particle continuum.

\section{DISCUSSION}

As noted previously, the argument that in the regime where Coulomb repulsion can be neglected attractive potentials for identical charge particles will lead to bound states in magnetic fields is quite general. However, getting into the regime in which Coulomb repulsion is negligible requires extraordinarily large magnetic fields. We see from Eq. (3.9) that unless there is fine tuning of the scattering length, the Coulomb correction becomes small when $\left(Q^{2}(\mu R) \frac{r_{0}^{2}}{R^{2}}\right) \ll 1$. A quick and dirty estimate for systems of atomic ions (with $R$ of order the Bohr radius and $\mu \sim M_{p}$ gives $B_{0} \gg 10^{11} \mathrm{~T}$. Clearly this is far beyond the regime for which the ions are essentially unaltered from free space and the interaction between the atoms is given by its free space value. The situation only worsens for systems of molecular ions.

For this reason it is apparent that if the phenomenon occurs in a clean system it is most likely to be for strongly interacting particles. The diproton system is particularly favorable since the scattering length is unnaturally large. From Eq. (4.6), it is apparent that one requires magnetic fields of order $10^{12} \mathrm{~T}$ for the Coulomb repulsion to be modest enough to be treated as a correction. This scale is of physical interest. Magnetic fields of this scale are believed to be found in nature - in particular on the surface of magnetars. Thus the novel type of bound state discussed in this paper appears to be physically relevant.

One important open issue associated with this phenomenon for the diproton is whether there is a maximum magnetic field for which the system binds. As one increases the magnetic field there are corrections to the effective field theory type treatment arising from shorter distance effects; assuming such corrections are modest, the scale of these corrections are given in Eq. (4.5). As $B_{0}$ is increased further, however, these corrections will become large and the expansion will break down. In principle one can simply solve the problem using "realistic" nuclear potentials. However, as $B_{0}$ increases, $r_{0}$ decreases. When $r_{0}$ becomes short enough that it is similar to the spatial extent of the nucleon one expects that magnetic effects would begin to cause substantial modifications of the nucleon substructure; this in turn could lead to substantial modifications of the nucleon-nucleon potential. Since the spatial extent of the nucleon is very similar to the range of the nucleon-nucleon potential it is 
not clear whether an explicit calculation with a realistic potential would provide any reliable information beyond what can be studied via the effective theory here.

Another important open question is whether there are any other strongly interacting systems besides the diproton for which this novel class of bound state will form. As noted in Sect. III] for systems with "natural" scattering lengths of order $R$ it appears that typical nuclear systems are marginal at best. If the field is strong enough to overcome Coulomb repulsion, the system may well be out of the weak field limit. As the field increases beyond this limit, the magnetic interactions may alter the internal structure of the nuclei altering the potentials. Thus, apart from diprotons in magnetar strength fields we know of no other clean examples of the phenomenon.

In looking for other systems, it should be noted that this paper only considers the case for which the system consists of identical charged particles; this leads to a decoupling of the center of mass and relative motion. While this simplifies the problem both conceptually and computationally, it also forces us to consider very strong magnetic fields to overcome the effects of the Coulomb repulsion. One might wish to consider the situation in which a charged particle and a neutral particle interact in the presence of a magnetic field. In such a situation, Coulomb repulsion would no longer be an issue. However, the notion of a two particle bound state becomes a bit more involved in the presence of a magnetic field since the relative and center-of-mass motion are coupled. In practice, a useful definition is that the two particles are in an energy eigenstate with the property that the position of the two particles are strongly correlated-if one observes one of the particles at some point, then the probability of not finding the other particle a distance $r$ away decreases exponentially with $r$ for large $r$ in any direction. An alternative definition is that there exists a state with an energy less than the lowest Landau level for the isolated charged particle. The simple qualitative arguments as to why one expects such bound states $a$ priori are weakened as compared to the case of identical charged particles; we do not know whether magnetic fields can cause a charged and neutral particle acting through a potential to bind (in the sense defined above) when they do not bind in free space. We will consider this possibility in a subsequent work.

Finally, we turn to the implications of this result from the astrophysical perspective. On the one hand, the magnetic field strength in magnetars appears to be large enough for bound diprotons to form. An optimistic view on this would be that this can have important effects on the dynamics of the magnetar leading to some observational signature. On the other hand, as discussed in Sect. V the bound diprotons are not the ground state of the system; spin aligned unbound protons are. To the extent that protons will tend to stay in their ground state, this suggests that it will be difficult to find an observational signature of bound diprotons. Our results, however, suggest that nuclei that are unstable due to excessive number of protons may become stable in the presence of a magnetic field of this magnitude. This shift of the nuclear valley of stability towards higher values of $Z$ deserves further study.
[1] D. B. Kaplan, arXiv:nucl-th/0510023

[2] C. P. Burgess, Phys. Rept. 330, 193 (2000) arXiv:hep-th/9808176.

[3] L. D. Landau and E. M. Lifshitz, Quantum Mechanics, Pergamon (1976).

[4] J. J. de Swart, C. P. F. Terheggen and V. G. J. Stoks, arXiv:nucl-th/9509032.

[5] See, for instance, P. F. Bedaque and U. van Kolck, Ann. Rev. Nucl. Part. Sci. 52, 339 (2002)
arXiv:nucl-th/0203055.

[6] U. van Kolck, Prog. Part. Nucl. Phys. 43, 337 (1999) arXiv:nucl-th/9902015.

[7] S. R. Beane, P. F. Bedaque, W. C. Haxton, D. R. Phillips and M. J. Savage, arXiv:nucl-th/0008064.

[8] D. R. Phillips, Czech. J. Phys. 52, B49 (2002) arXiv:nucl-th/0203040. 